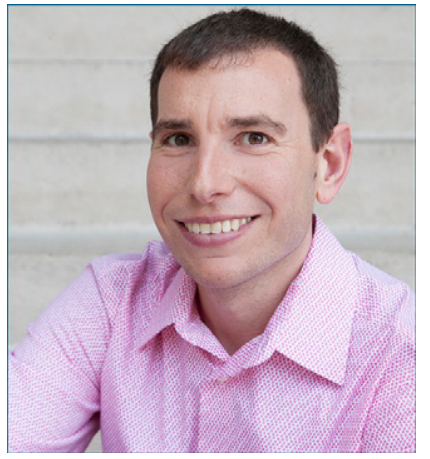

DANIEL L. HERTZ, PharmD, PhD

Daniel L. Hertz, PharmD, PhD, is an Assistant Professor in the University of Michigan College of Pharmacy and a member of the University of Michigan Rogel Cancer Center. Dr. Hertz's research interest is developing tools for individualizing treatment in patients with cancer and translating them into clinical practice, with a particular focus in taxaneinduced peripheral neuropathy. doi: 10.6004/jnccn.2019.7527

The ideas and viewpoints expressed in this commentary are those of the author and do not necessarily represent any policy, position, or program of NCCN.

\section{Including DPYD on Cancer Genetic Panels to Prevent Fatal Fluoropyrimidine Toxicity}

\author{
Daniel L. Hertz, PharmD, PhD, and Vaibhav Sahai, MBBS, MS
}

5 -fluorouracil (5-FU) and its oral prodrug capecitabine are fluoropyrimidine agents recommended within NCCN Clinical Practice Guidelines in Oncology (NCCN Guidelines) for many solid tumors, including colon, ${ }^{1}$ rectal, ${ }^{2}$ pancreatic, ${ }^{3}$ biliary tract, ${ }^{4}$ gastric, ${ }^{5}$ head and neck, ${ }^{6}$ ovarian, ${ }^{7}$ and breast cancer. ${ }^{8}$ These agents have generally acceptable toxicity profiles, but a subset of patients experience severe, sometimes fatal toxicity, including myelosuppression, gastrointestinal toxicity, mucositis, and hand-foot syndrome. Severe toxicity is due in part to inherited polymorphisms in dihydropyrimidine dehydrogenase, encoded by the DPYD gene, which is the enzyme responsible for fluoropyrimidine elimination. A meta-analysis of $>5,000$ patients validated 4 decreasedactivity DPYD polymorphisms (DPYD*2A [rs3918290], DPYD*13 [rs55886062]، DPYD D949V [rs67376798], and DPYD HapB3 [rs56038477]) that increase severe toxicity (adjusted relative risk for carrying a variant: $1.59-4.30) .{ }^{9}$ Approximately $7 \%$ of Caucasian patients carry 1 of these 4 DPYD variants, ${ }^{10}$ which confer $>50 \%$ risk of severe toxicity ${ }^{9}$ and approximately $3 \%$ risk of fatal toxicity ${ }^{11}$ in patients receiving standard fluoropyrimidine dosing. The NCCN Guidelines for Colon Cancer state that carriers of certain DPYD variants "have a significantly elevated risk for severe, life-threatening toxicity after a standard dose of fluoropyrimidine"1; however, NCCN Guidelines that recommend fluoropyrimidine agents for other tumor types do not include such a statement. ${ }^{2-8}$

The increased risk of life-threatening toxicity for variant carriers, so-called clinical validity, is necessary but not sufficient for recommending preemptive DPYD genetic testing. The recommendation to test requires clinical utility or evidence that genotyping improves clinical outcomes, which is most directly demonstrated in prospective clinical trials that randomize patients to DPYD-guided treatment or non-DPYD-guided standard of care..$^{12}$ The ideal prospective randomized controlled trial has not been conducted, but the NCCN Guidelines for Colon Cancer acknowledge that, "Two prospective studies have shown DPYD genotyping and fluoropyrimidine dose individualization to be feasible in clinical practice, improve patient safety, and be cost-effective." ${ }^{1}$

Deenen et al ${ }^{13}$ genotyped DPYD*2A in 1,631 fluoropyrimidine-treated patients and dose-reduced $D P Y D^{\star} 2 A$ carriers by $50 \%$, followed by dose escalation as tolerated. The rate of grade $\geq 3$ toxicity was significantly reduced in $D P Y D^{\star} 2 A$ carriers receiving dose reduction ( 5 of $18 ; 28 \%$ ) compared with historical controls receiving standard doses (35 of 48; 73\%; $P<.001) .{ }^{13}$ In another study, 1,181 patients were screened for the 4 validated DPYD variants, followed by a $25 \%$ or $50 \%$ dose reduction, depending on which variant was present. ${ }^{14}$ Similar to the findings from Deenen et al, $50 \%$ dose reduction in $D P Y D^{\star} 2 \mathrm{~A}$ carriers normalized the rates of grade $\geq 3$ toxicity (31\%), but $25 \%$ dose reductions did not sufficiently reduce toxicity in carriers of D949V (47\%) or HapB3 (39\%), suggesting larger dose reductions are necessary. Across the 2 trials, 0 of $98(0 \%)$ DPYD variant carriers who received reduced doses experienced fatal toxicity compared with 1 of 4 (25\%) variant carriers who were inadvertently treated with standard doses. Based on these prospective studies, dosing guidelines from the Clinical Pharmacogenetics Implementation Consortium recommend carriers of these 4 DPYD 
polymorphisms receive an initial $50 \%$ dose reduction followed by dose escalation as tolerated. ${ }^{10}$

Despite these demonstrations of clinical benefit, clinical uptake of preemptive DPYD genotyping has been limited. One reason for the lack of clinical uptake is that the benefit in reducing rates of severe (from $>50 \%$ to approximately $30 \%$ ) and fatal (from approximately $3 \%$ to $<1 \%$ ) toxicity in DPYD carriers must be weighed against the potential risk for diminished efficacy, particularly in the up to $50 \%$ of DPYD carriers who would not have experienced severe toxicity and received unnecessary dose reduction. These concerns are somewhat attenuated by the similar toxicity rates and systemic drug concentrations in DPYD*2A carriers receiving $50 \%$ dose reduction compared with noncarriers receiving standard doses ${ }^{13}$ and the recommendation to dose-escalate based on tolerability. ${ }^{10}$ However, noninferior efficacy has not been formally shown in a randomized prospective clinical trial, which could be the reason that the NCCN Guidelines for Colon Cancer state, "Universal pretreatment DPYD genotyping remains controversial, however, and the NCCN Panel does not support it at this time," 1 and that oncology clinical practice guidelines from $\mathrm{ASCO}^{15}$ and $\mathrm{ESMO}^{16}$ do not recommend DPYD testing.

The primary intent of this commentary is not to advocate for preemptive DPYD genotyping, which has been extensively reviewed and debated elsewhere. Although DPYD genotyping reduces overall healthcare $\operatorname{costs}^{13}$ due to reductions in toxicity-related hospitalization and use of the expensive rescue medication uridine triacetate, ${ }^{17}$ one of the barriers to clinical uptake is likely to be the upfront cost and inconvenience of DPYD genetic testing. Rather, our intent with this commentary is to advocate for including DPYD within genetic testing that is otherwise being conducted in patients with cancer, as a highly efficient strategy to identify DPYD carriers without incurring the cost or inconvenience of additional testing in all patients. Clinicians can then decide whether their patients should be considered for initial dose reduction based on the established decrease in toxicity, or receive standard doses based on the potential concern for decreased efficacy.

Patients with cancer undergo genetic testing for several reasons. Testing of the tumor's somatic genome for treatment selection or trial enrollment often includes matched germline genotyping to differentiate somatic mutations from germline polymorphisms and/or to identify inherited cancer susceptibility syndromes. DPYD should be included in matched germline testing conducted simultaneously with somatic tumor genetic testing. ${ }^{18}$ When matched germline testing is not conducted, DPYD should be included in somatic testing, followed by confirmatory germline testing in a CLIA-validated laboratory to exclude the unlikely possibility of a somatic mutation mimicking the germline polymorphism. Although this approach would incur the cost and inconvenience of DPYD testing for that patient, confirmatory testing of only the approximately $7 \%$ of suspected DPYD carriers is much more efficient than testing all patients before treatment.

The NCCN Guidelines recommend germline genetic testing for selected patients with colorectal, breast, and ovarian cancers, and all patients with pancreatic cancer. ${ }^{1,3,7,8}$ These are tumor types for which NCCN Guidelines include fluoropyrimidine regimens. Patients with these tumor types have a $5 \%$ to $10 \%$ prevalence of an actionable germline mutation, which is similar to the approximately $7 \%$ prevalence of carrying a potentially actionable DPYD allele. NCCN Guidelines for Genetic/Familial High-Risk Assessment: Breast, Ovarian, and Pancreatic recommend consideration of multigene panel testing for certain patients who meet testing criteria. ${ }^{19}$ Multigene panel testing may include intermediate penetrant genes without clinical actionability.

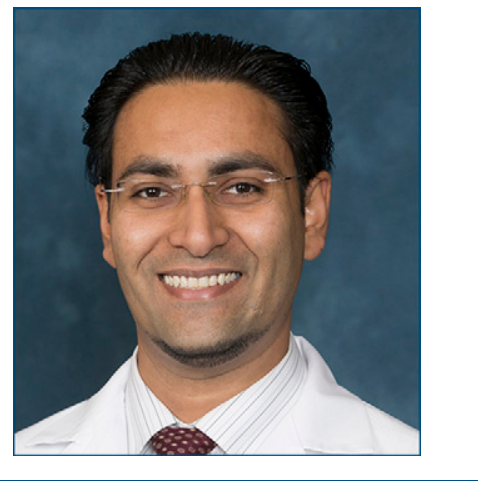

VIABHAV SAHAI, MBBS, MS

Vaibhav Sahai MBBS, MS, is an Associate Professor of Internal Medicine and Section Lead of the Gastrointestinal Oncology in the Division of Hematology-Oncology at University of Michigan. Dr. Sahai sees patients within the Multidisciplinary Pancreatic and Liver Tumor programs. As a clinical investigator he is the Principal Investigator on several investigator-initiated clinical trials open across the United States. 
DPYD should be included on all multigene germline panels for patients with tumor types that receive fluoropyrimidines, and is currently included on the Color panel (www.color.com). ${ }^{20}$ Finally, patients suspected of carrying a DPYD variant based on nonclinical, direct-to-consumer genetic testing such as 23andMe, which includes DPYD variants, should also be sent for confirmatory germline DPYD testing in a CLIA-validated laboratory before fluoropyrimidine treatment.

Testing for 4 reduced-activity polymorphisms in $D P Y D$ decreases incidence of severe toxicity from fluoropyrimidine treatment but is not standard practice due to concerns about the potential for decreased efficacy, costs and inconvenience of testing, and lack of endorsement from oncology guidelines. We advocate for inclusion of DPYD within genetic testing otherwise being conducted in patients with cancer types treated with fluoropyrimidine agents. We believe this represents a highly efficient strategy to identify DPYD carriers for whom fluoropyrimidine dose reduction should be considered to prevent severe, sometimes fatal, toxicity.

Disclosures: The authors have disclosed that they have not received any financial considerations from any person or organization to support the preparation, analysis, results, or discussion of this article.

Correspondence: Daniel L. Hertz, PharmD, PhD, Department of Clinical Pharmacy, University of Michigan College of Pharmacy, 1100 North University Building, Room 2560C, Ann Arbor, Ml 48109-1065. Email: DLHertz@med.umich.edu

\section{References}

1. Benson AB III, Venook AP, Al-Hawary M, et al. NCCN Clinical Practice Guidelines in Oncology: Colon Cancer. Version 1.2020. Accessed February 21, 2020. To view the most recent version, visit NCCN.org.

2. Benson AB III, Venook AP, Al-Hawary M, et al. NCCN Clinical Practice Guidelines in Oncology: Rectal Cancer. Version 1.2020. Accessed February 21, 2020. To view the most recent version, visit NCCN.org.

3. Tempero MA, Malafa MP, Al-Hawary M, et al. NCCN Clinical Practice Guidelines in Oncology: Pancreatic Adenocarcinoma. Version 1.2020. Accessed February 21, 2020. To view the most recent version, visit NCCN.org.

4. Benson AB III, D'Angelica MI, Abbott DE, et al. NCCN Clinical Practice Guidelines in Oncology: Hepatobiliary Cancers. Version 4.2019. Accessed February 21, 2020. To view the most recent version, visit NCCN.org.

5. Ajani JA, D'Amico TA, Bentrem DJ, et al. NCCN Clinical Practice Guidelines in Oncology: Gastric Cancer. Version 4.2019. Accessed February 21, 2020. To view the most recent version, visit NCCN.org.

6. Pfister DG, Spencer S, Adelstein D, et al. NCCN Clinical Practice Guidelines in Oncology: Head and Neck Cancers. Version 1.2020. Accessed February 21, 2020. To view the most recent version, visit NCCN.org.

7. Armstrong DK, Alvarez RD, Bakkum-Gomez, et al. NCCN Clinical Practice Guidelines in Oncology: Ovarian Cancer. Version 3.2019. Accessed February 21, 2020. To view the most recent version, visit NCCN.org.

8. Gradishar WJ, Anderson BO, Abraham J, et al. NCCN Clinical Practice Guidelines in Oncology: Breast Cancer. Version 3.2020. Accessed February 21, 2020. To view the most recent version, visit NCCN.org.

9. Meulendijks D, Henricks LM, Sonke GS, et al. Clinical relevance of DPYD variants c. $1679 \mathrm{~T}>\mathrm{G}$, c. $1236 \mathrm{G}>\mathrm{A} / \mathrm{HapB} 3$, and c. $1601 \mathrm{G}>\mathrm{A}$ as predictors of severe fluoropyrimidine-associated toxicity: a systematic review and meta-analysis of individual patient data. Lancet Oncol 2015;16: 1639-1650.

10. Amstutz U, Henricks LM, Offer SM, et al. Clinical Pharmacogenetics Implementation Consortium (CPIC) guideline for dihydropyrimidine dehydrogenase genotype and fluoropyrimidine dosing: 2017 update. Clin Pharmacol Ther 2018;103:210-216.
11. Rai K, Batukbhai BDO, Brooks GA. Risk of treatment-related death in carriers of pathogenic DPYD polymorphisms treated with fluoropyrimidine chemotherapy: a systematic review and patient-level analysis [abstract]. J Clin Oncol 2019;37(Suppl):Abstract e15132.

12. Institute of Medicine. Evolution of Translational Omics: Lessons Learned and the Path Forward. Washington, DC: The National Academies Press; 2012.

13. Deenen MJ, Meulendijks D, Cats A, et al. Upfront genotyping of DPYD*2A to individualize fluoropyrimidine therapy: a safety and cost analysis. J Clin Oncol 2016;34:227-234.

14. Henricks LM, Lunenburg CATC, de Man FM, et al. DPYD genotypeguided dose individualisation of fluoropyrimidine therapy in patients with cancer: a prospective safety analysis. Lancet Oncol 2018;19:1459-1467.

15. Sepulveda AR, Hamilton SR, Allegra CJ, et al. Molecular biomarkers for the evaluation of colorectal cancer: guideline from the American Society for Clinical Pathology, College of American Pathologists, Association for Molecular Pathology, and the American Society of Clinical Oncology. $\mathrm{J}$ Clin Oncol 2017;35:1453-1486.

16. Labianca R, Nordlinger B, Beretta GD, et al. Early colon cancer: ESMO clinical practice guidelines for diagnosis, treatment and follow-up. Ann Oncol 2013;24(Suppl 6):vi64-72.

17. Toffoli G, Innocenti F, Polesel J, et al. The genotype for DPYD risk variants in patients with colorectal cancer and the related toxicity management costs in clinical practice. Clin Pharmacol Ther 2019;105:994-1002.

18. Hertz DL, Glatz A, Pasternak AL, et al. Integration of germline pharmacogenetics into a tumor sequencing program [published online July 23 2018]. JCO Precis Oncol, doi: 10.1200/PO.18.00011

19. Daly MB, Pilarski R, Berry MP, et al. NCCN Clinical Practice Guidelines in Oncology: Genetic/Familial High-Risk Assessment: Breast, Ovarian, and Pancreatic. Version 3.2019. Accessed June 27, 2019. To view the most recent version, visit NCCN.org.

20. Neben $C L$, Zimmer AD, Stedden W, et al. Multi-gene panel testing of 23,179 individuals for hereditary cancer risk identifies pathogenic variant carriers missed by current genetic testing guidelines. J Mol Diagn 2019; 21:646-657. 